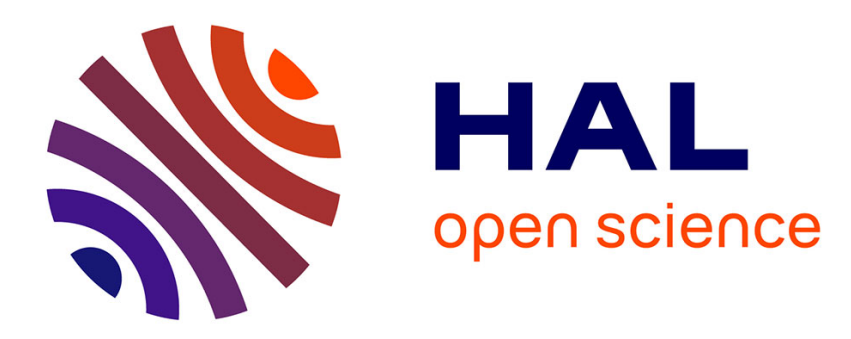

\title{
Les troubles psychotiques post-traumatiques : un modèle physiopathologique de la schizophrénie
}

\author{
Y. Auxéméry
}

\section{To cite this version:}

Y. Auxéméry. Les troubles psychotiques post-traumatiques : un modèle physiopathologique de la schizophrénie. Annales Médico-Psychologiques, Revue Psychiatrique, 2011, 169 (3), pp.155. 10.1016/j.amp.2009.12.020 . hal-00739339

\section{HAL Id: hal-00739339 \\ https://hal.science/hal-00739339}

Submitted on 8 Oct 2012

HAL is a multi-disciplinary open access archive for the deposit and dissemination of scientific research documents, whether they are published or not. The documents may come from teaching and research institutions in France or abroad, or from public or private research centers.
L'archive ouverte pluridisciplinaire HAL, est destinée au dépôt et à la diffusion de documents scientifiques de niveau recherche, publiés ou non, émanant des établissements d'enseignement et de recherche français ou étrangers, des laboratoires publics ou privés. 


\section{Accepted Manuscript}

Title: Les troubles psychotiques post-traumatiques : un modèle physiopathologique de la schizophrénie

Author: Y. Auxéméry

PII: $\quad$ S0003-4487(10)00281-7

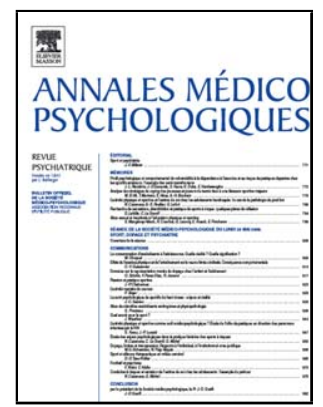

DOI: $\quad$ doi:10.1016/j.amp.2009.12.020

Reference: $\quad$ AMEPSY 1231

To appear in: $\quad$ Annales Médico-Psychologiques

Received date: $\quad 21-10-2009$

Accepted date: $\quad 8-11-2009$

Please cite this article as: Auxéméry Y, Les troubles psychotiques post-traumatiques : un modèle physiopathologique de la schizophrénie, Annales medio-psychologiques (2010), doi:10.1016/j.amp.2009.12.020

This is a PDF file of an unedited manuscript that has been accepted for publication. As a service to our customers we are providing this early version of the manuscript. The manuscript will undergo copyediting, typesetting, and review of the resulting proof before it is published in its final form. Please note that during the production process errors may be discovered which could affect the content, and all legal disclaimers that apply to the journal pertain. 


\section{Mémoire}

Les troubles psychotiques post-traumatiques : un modèle physiopathologique de la schizophrénie

Posttraumatic psychotic symptoms: A physiopathological model of schizophrenia Y. Auxéméry

Service de psychiatrie et de psychologie clinique, Hôpital d'Instruction des Armées Legouest, 27 Avenue de Plantières, BP 90001, Metz cedex 3, France

Texte reçu le 21 octobre 2009 ; accepté le 8 novembre 2009

\section{Résumé}

La schizophrénie et le traumatisme crânien sont deux problèmes majeurs de santé publique. Parmi les complications neuropsychologiques et psychiatriques secondaires au traumatisme crânien, les psychoses post-traumatiques interrogent plusieurs cadres nosographiques. Le trouble psychotique secondaire à une affection médicale générale nécessite d'affirmer une étiologie physiologique directe entre l'affection médicale traumatique et les symptômes psychotiques. La Schizophrenia-like psychosis est un syndrome schizophrénique secondaire au traumatisme crânien et qui traduit une atteinte cérébrale diffuse. Cette entité clinique est différenciée de la schizophrénie post-traumatique qui désigne une schizophrénie primaire décompensée dans les suites d'un traumatisme crânien.

Ces différents diagnostics catégoriels peuvent être réunis au sein d'une approche dimensionnelle des symptômes psychotiques post-traumatiques. Un tel continuum clinique interroge les théories physiopathologiques de la schizophrénie dans une approche étiologique. Les modèles neurodégénératif, de dysrégulation dopaminergique et neurodéveloppemental sont utiles pour expliciter les troubles psychotiques post-traumatiques. Dans le même temps, la physiopathologie de la schizophrénie primaire apporte des éléments de compréhension étiologique pour les troubles psychotiques post-traumatiques. Dans les deux cas, une vulnérabilité génétique éprouvée par le facteur traumatique prend une expression clinique psychotique. Certains troubles psychotiques post-traumatiques sont à considérer comme des formes phénotypiques particulières du spectre de la schizophrénie. L'interaction entre le patrimoine génétique du sujet et l'environnement extérieur met en jeu les mécanismes de neuroplasticité cérébrale. Les avancées techniques de neuroimagerie (calculs volumétriques, 
calculs de densité cellulaire) offrent des perspectives d'études pour la compréhension neurophysiologique des troubles psychotiques post-traumatiques en lien avec la schizophrénie primaire.

Mots clés : Modèle neurodéveloppemental ; Physiopathologie ; Psychose post-traumatique ; Schizophrénie post-traumatique ; Symptômes psychotiques ; Traumatisme crânien

\section{Abstract}

Traumatic Brain Injury (TBI) and later serious psychopathology have long been associated in the literature. Psychotic disorder following traumatic head injury is reported to occur in 0,7 to $8,9 \%$ of patients who sustain a head injury. This paper summarizes the recent literature about psychotic symptoms following traumatic brain injury (TBI) and try to articulate these psychotic disorders to a pathophysiological model of primary schizophrenia.

Psychotic disorder due to a general medical condition (TBI) indicates that the hallucinations or delusions are the direct physical consequences of the medical condition. A contrario, schizophrenia-like psychosis is a secondary schizophreniform syndrom that must be differentiated from post-traumatic schizophrenia where the head trauma is an external factor furthering the onset of a primary psychosis. A dimensional approach could although take place for understanding pathophysiology.

Head injury has been reported to increase the likelihood of the development of psychosis disorder due to TBI, schizophrenia-like psychosis and posttraumatic schizophrenia. While TBI is a major public health issue, schizophrenia following TBI is relatively rare and poorly studied. The onset of schizophrenia occurs most commonly from late adolescence to midadulthood, in a age group where head injury is more frequent. Even if early illness features of schizophrenia might increase exposure to TBI, posttraumatic schizophrenia could be the result of a gene-environment interaction.

The pathophysiology research in psychotic disorders following traumatic brain injury don't must distinguish schizophrenia from other psychotic syndromes but have to consider a dimensional approach of these psychotic phenomena. Family history of schizophrenia and frontal or parietal lobe deficits were more common in patients with posttraumatic psychotic symptoms.

Susceptibility to schizophrenia is believed to be due to multiple genetic and interacting factors and mild childhood head injury may play a role in the development and onset of schizophrenia in families with a strong genetic predisposition. 
The authors suggest hypotheses aimed at furthering the understanding of the physiologic mechanisms relating traumatic brain injuries to psychotic symptoms. Psychotic disorder following TBI, schizophrenia like psychosis and posttraumatic schizophrenia are a potential interest to clinicians and neuroscientists, as it may provide clues to understanding primary psychotic disorders such as schizophrenia.

Schizophrenia is a psychotic disorder commonly attributed to the interaction of genetic vulnerability and environmental events which implies that environmental factors modulate the effects of the genotype. Schizophrenia related to TBI could also be the result of a geneenvironment interaction. Schizophrenia genes may increase exposure to head trauma (through agitation or cognitive impairment), with head trauma further increasing the risk for schizophrenia.

Further studies are needed to articulate the links between secondary psychotic symptoms and primary schizophrenia in a structure/function paradigm. The new imaging techniques of magnetic resonance imaging, position emission tomography and single-photon emission computed tomography could aid in this sense.

Keywords: Neurodevelopmental Model; Pathophysiology; Psychotic Symptoms; Posttraumatic Psychosis; Posttraumatic Schizophrenia; Traumatic Brain Injury

«Les blessures du crâne produisent le délire » Hippocrate, Aphorismes, Section VII ; 24.

\section{Introduction}

La schizophrénie et le traumatisme crânien (TC) sont deux problèmes majeurs de santé publique. La schizophrénie se déclare habituellement entre l'adolescence et le début de l'âge adulte pour atteindre une prévalence de près de $1 \%$ de la population générale. Le traumatisme crânien, surreprésenté chez les jeunes, concerne plus de 150000 français chaque année [29]. Les liens épidémiologiques entre TC et schizophrénie sont difficiles à établir. Les patients schizophrènes présentent davantage de TC que la population générale [44] peut-être $\mathrm{du}$ fait d'incoordination motrice et/ou de troubles du comportement. Par ailleurs les antécédents déclarés de TC chez des patients psychotiques peuvent être sous-évalués du fait de troubles cognitifs ou au contraire surévalués comme rationalisation $\mathrm{du}$ trouble 
psychiatrique. Seules quelques études rétrospectives ont utilisé des données d'archives médicales pour authentifier la réalité du TC chez ces patients psychotiques.

Les complications neuropsychologiques et psychiatriques secondaires à un TC sont nombreuses et handicapantes [15]. Si la dépression et les troubles anxieux sont les troubles psychiatriques les plus fréquents, le taux de psychose post-traumatique est très variable en fonction des études allant de 0,7 à $9 \%$ des cas $[2,10,12]$. La terminologie non consensuelle utilisée par les auteurs entre les différents cadres nosographiques retenus tant au niveau de la définition psychotique (des symptômes psychotiques à la schizophrénie) que du traumatisme crânien (les multiples atteintes céphaliques traumatiques) rend difficile la comparaison des travaux effectués sur le thème.

La question des liens entre symptômes psychotiques (schizophréniques ou non) et TC est complexe. Elle peut éclairer la physiopathologie de la schizophrénie. Après un bref historique nous envisagerons successivement le trouble psychotique secondaire à un TC, la Schizophrenia-like psychosis et la schizophrénie post-traumatique. Les symptômes psychotiques post-traumatiques interrogent les modèles dopaminergique, dégénératif et neuro-développemental de la schizophrénie.

\section{Historique}

Bleuler évoque la notion de schizophrénie secondaire à un traumatisme crânien avec une présentation et une évolution clinique identique à la schizophrénie idiopathique. Kraepelin décrit que les traumatismes crâniens dans l'enfance sont des facteurs prédisposant à la démence précoce. Au sortir de la Grande Guerre, Schneider rapporte la Kriegpsychose chez trois observations cliniques de soldats ensevelis [37]. S'il focalise son intérêt sur la frayeur ressentie par le sujet comme facteur déclenchant la symptomatologie psychotique, nous savons aujourd'hui que le vent du boulet est aussi source de commotion cérébrale.

La question de la schizophrénie post-traumatique est régulièrement évoquée par la Société Médico-Psychologique. Dès 1932, Schultz note que le traumatisme crânien possède un rôle facilitateur du déclenchement psychotique [38], position confirmée par Ey et Cornavin après la Deuxième Guerre mondiale [14]. En 1967 Alliez et Sormani réalisent une revue de la littérature regroupant plus de trente observations de psychoses post-traumatiques [3]. La différenciation est difficile entre des symptômes psychotiques schizophréniformes lésionnels d'une blessure cérébrale et une schizophrénie primaire déclenchée par le TC. Un autre auteur, De Morsier, évoque la schizophrénie traumatique et affirme l'existence d'un facteur 
étiologique direct entre le TC et le syndrome hallucinatoire [13]. Si Senninger définit clairement la schizophrénie post-traumatique [40], a contrario Léger et al. retiennent des manifestations psychotiques post-traumatiques transitoires sans nécessaire schizophrénie caractérisée [26].

Borras et al. abordent la question sur un versant étiologique dans une communication récente à la Société Médico-Psychologique. Ils suggèrent que les «troubles psychotiques survenant dans le décours d'un traumatisme crânio-cérébral » peuvent entrer dans un modèle d'interactions complexes entre gènes et facteurs environnementaux [7].

\section{Des symptômes psychotiques post-traumatiques isolés à la schizophrénie post- traumatique : vers une approche dimensionnelle}

Un traumatisme crânien grave et ses conséquences somatiques directes - ou retardées - sur le cerveau peuvent être pourvoyeurs de symptômes psychotiques. Les mécanismes lésionnels sont très divers : accélération-décélération, atteintes vasculaires ischémiques et hémorragiques, hypertension intracrânienne chronique, embarrures, plaies crânio-cérébrales, hématomes intracrâniens... Il s'agit ici de trouble psychotique induit par une affection neurologique séquellaire d'un traumatisme crânio-cérébral. Les symptômes psychotiques résultants peuvent être isolés et ne pas correspondre à un syndrome psychiatrique précis. Mais plusieurs symptômes psychotiques peuvent aussi s'associer pour donner un tableau schizophrénique dénommé Schizophrenia-like psychosis. Nous envisagerons successivement les symptômes psychotiques isolés et la Schizophrenia-like psychosis avant de discuter la présentation psychotique du syndrome subjectif des traumatisés crâniens. Dans un second temps nous redéfinirons la schizophrénie post-traumatique, schizophrénie déclenchée par un traumatisme crânien.

\subsection{Troubles psychotiques induits par le traumatisme crânien}

Dans le trouble psychotique secondaire à un traumatisme crânien, l'étiologie $d u / d e s$ symptôme(s) psychotique(s) est directement liée à une atteinte cérébrale localisée en référence au paradigme structure-fonction. À une lésion cérébrale documentée à la neuroimagerie correspond un symptôme psychotique précis. Les hallucinations peuvent par exemple être causées par une épilepsie temporale séquellaire [31]. Au sens du DSM-IV-TR [4] les symptômes psychotiques doivent être la conséquence directe de l'affection médicale 
générale (ici le TC) à l'exclusion de tout trouble psychiatrique primaire, y compris dans les antécédents familiaux [17].

Le trouble psychotique induit par un TC ou psychose secondaire inclut souvent un délire de persécution et des hallucinations auditives en l'absence de symptôme négatif $[18,19]$. Dans une étude réalisée auprès d'anciens combattants finlandais traumatisés, près de $30 \%$ sont diagnostiqués comme présentant un délire psychotique alors que la schizophrénie paranoïde est retenue dans un peu moins de $15 \%$ des cas [2]. Des symptômes psychotiques post-traumatiques peuvent rester isolés ou s'organiser en syndrome. Signer décrit une observation de délire érotomaniaque secondaire à un TC [42]. Il est à noter qu'un syndrome maniaque post-traumatique avec caractéristiques psychotiques a été rapporté par plusieurs auteurs comme l'expression clinique de lésions corticales temporales et orbito-frontales $[23,35]$.

Parmi les syndromes psychotiques post-traumatiques, la Schizophrenia-like psychosis désigne un syndrome schizophrénique secondaire à un TC [10,41,49]. Cette entité clinique se caractérise par la prédominance de symptômes positifs avec des persécutions et des hallucinations auditives, contrastant avec le peu de symptômes négatifs [49]. Le facteur de risque le plus fidèle est la présence d'atteinte cérébrale diffuse et particulièrement des lobes temporaux et frontaux. Un syndrome dépressif prodromique est fréquent avant l'entrée progressive dans la pathologie psychotique qui se chronicise ensuite. Fujii analyse 70 cas de syndromes schizophréniformes après TC. Le sex-ratio est masculin. Le temps de latence entre le TC - souvent sévère - et le diagnostic de trouble délirant est de quatre ans en moyenne. Les auteurs notent $65 \%$ d'anomalies frontales ou temporales à la neuro-imagerie [18]. Dans une étude prospective, Sachdev note également un mode d'entrée progressif dans la Schizophrenia-like psychosis, avec un temps de latence de plus de quatre ans par rapport au TC [36].

Les syndromes psychotiques post-traumatiques sont à confronter au syndrome subjectif des traumatisés crâniens défini par Pierre Marie en 1916 et rebaptisé par les AngloSaxons syndrome post-commotionnel. Ce cadre diagnostique se caractérise par une absence de parallélisme anatomo-clinique entre un TC de faible intensité et des plaintes fonctionnelles invalidantes. Une réunion de la société française de neurologie au sortir de la Première Guerre mondiale offre une description précise du tableau clinique caractérisé par des symptômes subjectifs avec troubles de l'humeur, de la mémoire, du sommeil, des céphalées et des éblouissements [34]. Si un délire de revendication peut être présent, des symptômes psychotiques négatifs à type de repli autistique sont beaucoup plus fréquents. Il est probable 
que les troubles psychotiques post-traumatiques sont davantage diagnostiqués lorsque les symptômes psychotiques sont positifs que lorsque prédominent des symptômes négatifs. Ces derniers feront sans doute davantage retenir le diagnostic de syndrome subjectif des traumatisés crâniens.

\subsection{Schizophrénie post-traumatique}

La schizophrénie post-traumatique désigne une schizophrénie au sens du DSM-IV-TR [4] et qui s'est révélée au décours d'un traumatisme crânien. Le diagnostic de schizophrénie exclut une affection médicale générale à l'origine du tableau psychiatrique. Il n'y a donc pas de lésion cérébrale documentée à la neuroimagerie. Le traumatisme a été un facteur déclenchant, révélant ou précipitant une schizophrénie latente. Grâce à l'étude d'observations personnelles, Senninger a défini pour sa pratique expertale des critères caractérisant la schizophrénie post-traumatique [40]. Dans le recueil des antécédents du patient, aucun symptôme psychotique ne doit avoir été décelé avant le traumatisme crânien. La gravité traumatologique du choc doit être suffisante pour induire initialement un syndrome confusionnel. Une période de latence d'une durée d'un an est fréquente avant que le diagnostic de schizophrénie soit évoqué. Cette phase paucisymptomatique est parfois grevée de repli autistique et de bizarreries témoignant de l'émergence d'un syndrome dissociatif. Survient in fine un délire paranoïde qui intègre l'événement traumatique dans son système paralogique. Si le thème principal du délire est la persécution, le mécanisme à l'œuvre est le plus souvent interprétatif. Pour Senninger, le schéma évolutif clinique de la schizophrénie post-traumatique est le suivant: traumatisme crânien, confusion mentale, syndrome dissociatif puis délire paranoïde [40].

D'autres études ont permis d'affiner les caractéristiques sociodémographiques des patients souffrant de schizophrénie post-traumatique. Il s'agit le plus souvent de sujets jeunes [25]. De Mol retrouve des adultes de moins de 30 ans [12], alors que pour De Morsier l'âge de début serait de 38 à 48 ans [13]. Le sex-ratio est masculin, de l'ordre de un quart[13] à un sixième [25]. Lorsqu'un trouble de la personnalité (schizotypique ou schizoïde) antérieur est noté, il est considéré comme un autre facteur de risque. La durée de la période de latence est de deux ans pour la majorité des publications [38,40]. Certains voudraient être plus stricts en retenant la limite d'un an [12]. Pour De Morsier, la durée de cette latence serait impossible à définir [13]. Ces divergences temporelles s'expliquent par des approches différentes en fonction des auteurs, dans une logique d'investigation médico-légale ou étiologique. 
Deux tableaux cliniques différents de schizophrénie post-traumatique peuvent finalement être retenus. La forme classiquement décrite est une schizophrénie avec symptômes positifs où le délire est au premier plan. Mais il faut également évoquer une forme déficitaire de schizophrénie post-traumatique dominée par les symptômes négatifs et le repli autistique. Cette seconde présentation moins bruyante est probablement sous-diagnostiquée en clinique et moins retrouvée dans un cadre expertal.

A contrario certains auteurs réfutent l'existence d'une schizophrénie post-traumatique en considérant le TC comme prodrome du mode d'entrée dans la schizophrénie et non comme facteur déclenchant cette dernière [9]. Dans l'étude de Nielsen et al., seulement $8 \%$ des hommes et $3 \%$ des femmes schizophrènes avaient préalablement été hospitalisés pour un traumatisme crânien [33]. Le TC constituerait alors une contribution modeste au développement de la schizophrénie [33,43]. Dans une étude de cohorte sur près de 800000 sujets, Harrison conclut encore plus catégoriquement que le TC n'augmente pas le risque de schizophrénie en population générale [20]. La corrélation entre TC et schizophrénie existerait uniquement dans les familles prédisposées présentant des antécédents familiaux psychotiques. Le TC n'est pas un facteur de risque indépendant de la schizophrénie [24]: d'autres facteurs étiologiques qui interagissent entre eux sont nécessaires [43].

\section{Des symptômes psychotiques post-traumatiques aux modèles physiopathologiques de la schizophrénie primaire}

Les cadres nosographiques de psychose secondaire à un TC, de Schizophrenia-like psychosis et de schizophrénie post-traumatique peuvent éclairer certains aspects neurophysiologiques de la schizophrénie primaire. Nous évoquerons successivement les hypothèses neurodégénérative, dopaminergique et neuro-développementale que nous articulerons aux troubles psychotiques post-traumatiques. Ces différentes théories physiopathologiques peuvent se rejoindre au sein du paradigme de l'interaction gènes/environnement.

\subsection{Hypothèse dégénérative}

L'association des symptômes psychotiques avec des lésions des lobes cérébraux frontaux et temporaux est décrite par plusieurs auteurs [2,10,16,21]. Ces lésions cérébrales traumatiques diffuses sont localisées dans des zones cérébrales similaires aux lésions 
cérébrales diffuses de pathologies démentielles dégénératives. Kraepelin avait décrit les traumatismes crâniens dans l'enfance comme un facteurs de risque de dementia praecox. Plus récemment, Angelergues et Audisio ont évoqué l'existence de démence post-traumatique grevée de symptômes psychotiques [5]. Le rôle des TC répétés comme facteur de risque de démence pugilistique avec symptômes psychotiques est bien connu. Les patients souffrant de Schizophrenia-like psychosis ont davantage de dommage cérébral étendu que ceux ayant subit un TC sans psychose ultérieure.

Plusieurs études ont permis d'établir un lien neurophysiologique entre schizophrénie et maladie d'Alzheimer. La baisse des capacités de réserve cérébrale a été évoquée alternativement pour chacune de ces deux pathologies [27]. Parallèlement à l'étude de Malaspina [28], une autre étude retrouve que le mode d'entrée dans la maladie d'Alzheimer est plus précoce si un TC antérieur est présent [32]. Le TC peut entraîner une accumulation cérébrale de peptide amyloïde bêta pourvoyeuse de déficits cognitifs [22]. Le modèle interactif gène/environnement entre le traumatisme crânien qui éprouve une vulnérabilité allélique de l'Apolipoprotéïne E est décrit dans la physiopathologie de la maladie d'Alzheimer [32,47]. Le même modèle est transposable à la schizophrénie post-traumatique. Un allèle codant pour l'Apolipoprotéine E peut être associé à des formes particulières de schizophrénie en influençant l'expression phénotypique de la maladie lorsqu'un TC est intervenu [39]. La schizophrénie post-traumatique peut alors être considérée comme un soustype de schizophrénie.

\subsection{Hypothèse dopaminergique}

Le trouble psychotique secondaire à un traumatisme crânio-cérébral a été différencié de la schizophrénie par une présentation clinique différente d'une part, mais également par l'absence d'antécédent psychotique parmi les familles de ces patients [4,17]. Si ce cadre nosographique semble initialement - au sens du DSM-IV-TR -, être écarté de la schizophrénie caractérisée, cette position catégorielle reste toutefois à discuter. En effet, les patients ayant des troubles neurologiques préalables au TC sont davantage susceptibles de développer une psychose post-traumatique [17]. Si des troubles neurologiques entraînant une altération motrice peuvent favoriser les traumatismes, une prédisposition neurobiologique à la psychose n'est pas à exclure pour autant. La présence de symptômes psychotiques secondaires à un TC peut résulter d'une interaction entre la blessure cérébrale et une prédisposition neurobiologique ante-traumatique à la schizophrénie. L'efficacité des 
antipsychotiques dans les symptômes psychotiques post-traumatiques (et dans la schizophrénie) est un argument indirect suggérant le rôle d'une dysrégulation dopaminergique dans ces pathologies [18]. Ainsi les antipsychotiques atypiques sont indiqués en première ligne thérapeutique pour traiter les symptômes psychotiques post-traumatiques et ce quel que soit le cadre nosographique retenu. Bennouna [6] rapproche la théorie cholinergique dans les psychoses après TC et dans la schizophrénie pour proposer l'adjonction d'un inhibiteur de l'acétylcholine estérase à l'antipsychotique atypique. Il est à noter que les anticonvulsivants et antidépresseurs sont également utiles pour traiter les comorbidités [30].

\subsection{Hypothèse neuro-développementale}

Pour l'équipe de Sachdev, les meilleurs éléments prédictifs de Schizophrenia-like psychosis sont la perte de connaissance initiale et les antécédents familiaux de schizophrénie [36,49]. La question étiologique du rôle du TC dans le déclenchement d'une schizophrénie post-traumatique a incité plusieurs auteurs à étudier la vulnérabilité familiale à la psychose chez les parents de patients ayant développé une schizophrénie post-traumatique. Dans une étude rétrospective canadienne, Abdelmalik compare l'anamnèse et la sévérité de traumatismes crâniens survenus dans l'enfance (période de maturation cérébrale) chez 67 sujets schizophrènes à 102 enfants de mêmes parents dans des familles présentant une susceptibilité à la schizophrénie [1]. Dans des familles génétiquement prédisposées à la schizophrénie, le TC dans l'enfance (avant l'âge de dix ans) augmente le risque de schizophrénie de 2,35 fois. L'âge médian de déclenchement schizophrénique est de cinq ans plus tôt si un TC est présent dans les antécédents du patient. Le délai médian entre le TC et l'éclosion psychotique est de 12 ans [1]. Un traumatisme crânien d'intensité modéré joue un rôle dans la survenue de schizophrénie chez un sujet génétiquement prédisposé, en favorisant le déclenchement psychotique qui survient plus tôt dans la vie, soit cinq ans plus précocement. Les auteurs émettent l'hypothèse que plus le TC est d'intensité sévère (sans pour autant de lésion visibles à la neuroimagerie) plus la schizophrénie apparaît tôt.

Malaspina confirme ces résultats, ajoutant que l'antécédent de TC est plus élevé chez les patients schizophrènes de familles vulnérables à la schizophrénie que chez les patients schizophrènes issus de familles prédisposées à la bipolarité $[8,28]$. Indépendamment du diagnostic psychiatrique présenté, les sujets issus des familles susceptibles à la schizophrénie ont davantage de TC que ceux issus de familles susceptibles à la bipolarité. Les gènes de vulnérabilité familiale à la schizophrénie exposeraient au traumatisme crânien, entraînant un 
effet synergique dans le déclenchement schizophrénique lui-même favorisé par ce traumatisme.

Dans un modèle neuro-développemental, la schizophrénie est une maladie aux déterminants multifactoriels, interactions d'une vulnérabilité génétique éprouvée par des facteurs extérieurs [45]. Le TC est alors considéré comme facteur de risque environnemental de la schizophrénie [11]. Les lésions cérébrales microscopiques secondaires à un traumatisme crânien peuvent entraîner un remodelage neuronal induisant le développement d'un trouble schizophrénique chez un sujet vulnérable et prédisposé [46]. Ce n'est donc pas une lésion cérébrale localisée qui implique le développement ultérieur de la psychose, mais le remodelage neuronal diffus d'une zone cérébrale. La latence entre le TC extérieur et l'apparition des premiers symptômes cliniques s'explique alors par le délai nécessaire aux changements neuronaux. La neuroplasticité cérébrale est ici affectée par la présence du traumatisme crânien pour prendre un développement pathologique. La (ou les) schizophrénie(s) post-traumatique(s) sont à considérer comme des phénotype(s) particulier(s) du spectre de « la » schizophrénie qui inclut plusieurs formes anatomo-cliniques [45].

\section{Conclusions}

Les symptômes psychotiques secondaires à un traumatisme crânien intéressent plusieurs cadres nosographiques dont le trouble psychotique secondaire à un TC, la Schizophrenia-like psychosis et la schizophrénie post-traumatique. Ces entités distinctement décrites dans la littérature peuvent se redéfinir dans une approche dimensionnelle. Ces syndromes éclairent certains aspects neurophysiologiques de la schizophrénie primaire. Ils en interrogent les modèles dopaminergique, dégénératif et neuro-développemental. Une vulnérabilité génétique interagit avec l'évènement traumatique en entrainant un remodelage neuronal s'exprimant en clinique par le (ou les) symptôme(s) psychotique(s).

D'autres études sont nécessaires pour dégager du spectre de la schizophrénie une entité spécifique de schizophrénie post-traumatique. Les avancées techniques de neuroimagerie (calculs volumétriques, calculs de densité cellulaire) offrent des perspectives nouvelles pour la compréhension physiopathologique des troubles psychotiques posttraumatiques en lien avec la schizophrénie primaire.

\section{Conflit d'intérêt : à compléter}




\section{Références}

[1] AbdelMalik P, Husted J, Chow EW, Basset AS. Childhood head injury and expression of schizophrenia in multiply affected families. Arch Gen Psychiatry 2003;60(3):231-36.

[2] Achté K, Jarho L, Kyykkä T, Vesterinen E. Paranoid disorders following war brain damage. Preliminary report. Psychopathology 1991;24(5):309-15.

[3] Alliez J, Sormani J. Réflexions sur la schizophrénie post-traumatique. Ann Méd Psychol $1967 ; 125(1): 1-21$.

[4] American Psychiatric Association. DSM-IV-TR. Manuel diagnostique et statistique des Troubles mentaux, $4^{\mathrm{e}}$ édition, Texte révisé (Washington DC, 2000).

[5] Angelergues R, Audisio M. Troubles mentaux secondaires aux traumatismes crâniens. Paris; Masson: 1964. EMC, 37520 A 10,1-15.

[6] Bennouna M, Greene VB, Defranoux L. Théorie cholinergique dans les psychoses après un traumatisme crânien et dans la schizophrénie : un lien ? L'Encéphale 2007;33(4Pt1):61620.

[7] Borras L, Damsa C, Vidailhet P, Andreoli A, Bianchi De Micheli F. Schizophrénies et traumatisme crânio-cérébral : Post hoc, ergo proper hoc ? Ann Med Psychol 2004;162:78893.

[8] Corcoran C, Malaspina D. Traumatic brain injury as a risk factor for schizophrenia. Curr Psychiatr Review 2007;3:25-32.

[9] David AS, Prince M. Psychosis following head injury: A critical review. J Neurol Neurosurg Psychiatry 2005;6:53-60.

[10] Davison K, Bagley CR. Schizophrenia like psychoses associated with organic disorders of the central nervous system: A review of the literature. In: Herrington $\mathrm{RN}$, ed. $\mathrm{Br} \mathrm{J}$ Psychiatry Special Publications 1969;4:113-84.

[11] Demily C, Thibaut F. Facteurs de risque environnementaux à la schizophrénie. Ann Med Psychol 2008;166:606-11.

[12] De Mol J, Violon A, Brihaye J. Post traumatic schizophrenic bouts: With regard to 6 cases of traumatic schizophrenia. L'Encéphale 1982;8:17-24.

[13] De Morsier G. Les hallucinations survenant après les traumatismes crânio-cérébraux. La schizophrénie traumatique. Ann Med Psychol 1972;130:183-94.

[14] Ey H, Cornavin J. Psychoses et événements de guerre. Ann Med Psychol 1948;2(106):923.

[15] Fann JR, Burington B, Leonetti A, Jaffe K, Katon WJ, Thompson RS. Psychiatric illness following traumatic brain injury in an adult health maintenance organization population. Arch 
Gen Psychiatry 2004;6(1):53-61.

[16] Feinstein A, Ron M. A longitudinal study of psychosis due to a general medical (neurological) condition: Establishing predictive and construct validity. J Neuropsychiatry Clin Neurosci 1998;10(4):448-52.

[17] Fujii D, Ahmed I. Risk factors in psychosis secondary to traumatic brain injury. J Neuropsychiatry Clin Neurosci 2001;13(1):61-9.

[18] Fujii D, Ahmed I. Psychotic disorder following traumatic brain injury: A conceptual framework. Cogn Neuropsychiatry 2002;7(1):41-62.

[19] Fujii, D, Ahmed I. Characteristics of psychotic disorder due to traumatic brain injury: an analysis of case studies in literature. J Neuropsychiatry Clin Neurosci 2002:14(2):130-40.

[20] Harrison G, Whitley E, Rasmussen F, Lewis G, Dalman C, Gunnell D. Risk of schizophrenia and other non-affective psychosis among individuals exposed to head injury: case-control study. Schizophr Res 2006;88(1-3):119-26.

[21] Hillbom E. After-effects of brain-injuries. Research on the symptoms causing invalidism of persons in Finland having sustained brain-injuries during the wars of 1939-1940 and 19411944. Acta Psychiatr Scand Suppl 1960;35(142):1-195.

[22] Jellinger KA. Head injury and dementia. Curr Opin Neurol 2004;17(6):719-23.

[23] Jorge RE, Robinson RG, Arndt SV, Starkstein SE, Forrester AW, Geisler F. Depression following traumatic brain injury: A 1 year longitudinal study. J Affect Disord 1993;27(4):233-43.

[24] Kim E. Does traumatic brain injury predispose individuals to develop schizophrenia? Curr Opin Psychiatry 2008;21(3):286-9.

[25] Lavie M. Un traumatisme peut-il déclencher une psychose chronique de l'adulte ? Stress et Trauma 2007;7(1):11-16.

[26] Léger JM, Plougeaut C, Dumond JJ, Garoux R. Le concept de schizophrénie posttraumatique est-il reconnu et utilisé par les psychiatres français ? Ann Med Psychol 1992;150(6):429-36.

[27] Lye TC, Shores EA. Traumatic brain injury as a risk factor for Alzheimer's disease : a review. Neuropsychol Rev 2000;10(2):115-129.

[28] Malaspina D, Goetz RR, Friedman JH, Kaufmann CA, Faraone SV, Tsuang M. et al. Traumatic brain injury and schizophrenia in members of schizophrenia and bipolar disorder pedigrees. Am J Psychiatry 2001;158:440-446.

[29] Mathé JF, Richard I, Rome J. Santé publique et traumatismes crâniens graves. Aspects épidémiologiques et financiers, structures et filières de soins. Ann Fr Anesth Réanim 
2005;24(6):688-94.

[30] McAllister TW, Ferrel RB. Evaluation and treatment of psychosis after traumatic brain injury. NeuroRehabilitation 2002;17(4):357-368.

[31] McKenna PJ, Kane JM, Parrish K. Psychotic syndromes in epilepsy. Am J Psychiatry 1985;142(8):895-904.

[32] Nemetz PN, Leibson C, Naessens JM, Beard M, Kokmen E, Annegers JF, Kurland LT. Traumatic brain injury and time to onset of Alzheimer's disease : a population-based study. Am J Epidemiol 1999;149:32-40.

[33] Nielsen AS, Mortensen PB, O’Callaghan E, Mors O, Ewald H. Is head injury a risk factor for schizophrenia ? Schizophr Res 2002;55(1-2);93-8.

[34] Réunion de la société neurologique de Paris avec les représentants des centres neurologiques militaires de France et des Pays Alliés. Revue Neurologique 1916 (Avril Mai) ; XXIX (4-5).

[35] Robinson RG, Boston JD, Starkstein SE, Prince TR. Comparison of mania and depression after brain injury: Causal factors. Am J Psychiatry 1988;145(2):172-78.

[36] Sachdev P, Smith JS, Cathcart S. Schizophrenia-like psychosis following traumatic brain injury: A chart-based descriptive and case-control study. Psychol Med 2001;31(2):231-9.

[37] Schneider K. Schizophrene Kriegpsychose. Zeit Neurol 1918;(43):420-39.

[38] Schultz B. La schizophrénie traumatique. Ann Med Psychol 1939;99:1-2.

[39] Schürhoff F, Krebs MO, Szöke A, Loze JY, Goldberger C, Quignon V. et al. Apolipoprotein E in schizophrenia: A french association study and meta-analysis. Am J Med Genet B Neuropsychiatry Genet 2003;119B:18-23.

[40] Senninger JL. La schizophrénie post-traumatique. Rev Fr Dommage Corp 1987;13(2):131-37.

[41] Shapiro LB: Schizophrenic-like psychosis following head injuries. Illinois Medical Journal 1939;76:250-54 .

[42] Signer SF, Cummings JL. De Clerambault's syndrome in organic affective disorder. Two cases. Br J Psychiatry 1987;151:404-7.

[43] Silver JM, Kramer R, Greenwald S, Weissman M. The association between head injuries and psychiiatric disorders: Findings from the New Haven NIMH Epidemiologic Catchment Area Study. Brain Inj 2001;15(11):935-45.

[44] Smeltzer DJ, Nasrallah HA, Miller SC. Psychotic disorders, in Neuropsychiatry of Traumatic Brain Injury, edited by Silver JM, Yudofsky SC, Hales RE. Washington, DC, American Psychiatric Press, 1994, p. 251-83. 
[45] Tandon R, Keshavan MS, Nasrallah HA. Schizophrenia, «just the facts »: What we known in 2008, Part II. Epidemiology and etiology. Schizophr Res 2008;102(1-3):1-18.

[46] Thomas G, Genest P, Walter M, Cozic JY. Les concepts de neurodéveloppement et neuroplasticité pourraient-ils expliquer les troubles psychotiques post-traumatiques ? Ann Med Psychol 2009;55:36-9.

[47] Van Den Heuvel C, Thornton E, Vink R. Traumatic brain injury and Alzheimer's disease: A review. Prog Brain Res 2007;161:303-16.

[48] Violon A. Post-traumatic psychoses. Acta Neurochir Suppl (Wien) 1988;44:67-69.

[49] Zhang Q, Sachdev PS. Psychotic disorder and traumatic brain injury. Curr Psychiatr Rep 2003;5(3):197-201. 